\title{
Otto Blumenthal über David Hilbert
}

\author{
Tilman Sauer und David E. Rowe
}

Otto Blumenthals Hommage an David Hilbert erschien 1922 in einem Heft der Naturwissenschaften, das dem 60. Geburtstag des Göttinger Mathematikers gewidmet war [I]. Blumenthal war der erste Doktorand Hilberts, managing editor der von ihm herausgegebenen Mathematischen Annalen und bestens mit seinem wissenschaftlichen Werk vertraut. Mit dem Wiederabdruck von Blumenthals Beitrag in diesem Heft der Mitteilungen ehrt die DMV nicht nur Hilbert im Jahr seines 150. Geburtstags, sondern sie erinnert damit auch an den 1944 in Theresienstadt ums Leben gekommenen Blumenthal.

Blumenthals Darstellung des Hilbertschen Wirkens in ihrer offenen Sympathie für den Jubilar gibt auch heute noch eine treffende Einschätzung und einen guten Überblick. Dass die Charakterisierung der Hilbertschen Beiträge zur Physik in diesem Beitrag von 1922 eher knapp ausfiel, liegt zum Teil darin begründet, dass diesen Aspekten der Hilbertschen Tätigkeit im selben Heft der Naturwissenschaften ein eigenständiger Beitrag von Max Born gewidmet war [2]. Dessen ungeachtet ist die knappe Bemerkung über Hilberts Anteil an der Begründung der allgemeinen Relativitätstheorie eine recht zutreffende Charakterisierung dieses Aspekts des Hilbertschen Werkes:

Und es soll nicht vergessen werden, dass er als erster in seinen „Grundlagen der Physik“ die Einsteinsche allgemeine Relativitätstheorie in einem wesentlichen Punkte weiter gebracht hat: die Einführung des Hamiltonschen Prinzips und der Weltfunktion zur Verknüpfung der Gravitation und der elektromagnetischen Erscheinungen stammt von ihm.

Freilich hat die wissenschaftshistorische Forschung diesen Punkt ausführlich und kontrovers diskutiert und kommentiert. Wir erinnern hier nur an die wichtigsten Punkte.

Hilberts Phase der Beschäftigung mit der modernen Physik $[4,17]$ in den Jahren 1912 bis 1918 kulminierte in den Monaten Oktober und November 1915 in einer intensiven Parallelforschung von Hilbert und Albert Einstein $[14,15]$. Ausgangspunkt war die sogenannte „Entwurf“Theorie Einsteins von 1913, die im Wesentlichen alle Elemente der vollendeten allgemeinen Relativitätstheorie bereits enthielt: die zentrale Rolle des metrischen Tensors zur Repräsentation des Gravito-Inertialfeldes, die Repräsentation der felderzeugenden Quellen durch einen symmetrischen Energie-Impuls-Spannungstensor und die Aufstellung allgemein kovarianter Bewegungsgleichungen. Was noch fehlte, waren allgemein kovariante Feldgleichungen. Die von Einstein und Marcel Grossmann in ihrem ,Entwurf einer verallgemeinerten Relativitätstheorie und einer Theorie der Gravitation“ [10] stattdessen publizierten Feldgleichungen waren manifest nicht allgemein kovariant und brachen damit die angestrebte Dif- feomorphismenkovarianz der ganzen Theorie. Mit einem post hoc erdachten Argument, der sogenannten Lochbetrachtung, hatte Einstein sich dann davon überzeugt, dass solche allgemein kovarianten Gleichungen aus prinzipiellen physikalischen Gründen auch gar nicht in Frage kämen.

Auf Einladung Hilberts hatte Einstein im Juli 1915 Göttingen besucht und in einer Serie von Vorträgen seine Theorie vorgestellt. Danach folgte für beide Forscher eine Phase der intensiven Reflexion auf die Voraussetzungen, Schwierigkeiten und Perspektiven dieser Theorie. Rekonstruktionen der jeweiligen Überlegungen Hilberts und Einsteins im Herbst 1915 sind in der neueren Literatur mehrfach vorgeschlagen worden, müssen aber wohl alle aufgrund der unvollständigen Quellenlage ab einem gewissen Punkt spekulativ bleiben. Fest steht, dass Einstein im November 1915 in wöchentlichem Abstand vier Mitteilungen in den Sitzungsberichten der Preußischen Akademie der Wissenschaften publiziert [6-9]. In der ersten dieser Arbeiten kehrt er zur allgemeinen Kovarianz des Riemanntensors zurück, schlägt aber auf dessen Grundlage nur Feldgleichungen vor, die unter einer eingeschränkten, der unimodularen Transformationsgruppe kovariant sind [8]. Eine Woche später jedoch gibt er diese Einschränkung auf und schlägt den Riccitensor als Gravitationstensor vor [9]. Dieser Vorschlag hatte aber nun zur Konsequenz, dass der Energieimpulstensor spurfrei sein müsste, wie er es wäre, wenn alle Materie im Fundament elektromagnetischen Ursprungs wäre. Wiederum eine Woche später gelingt Einstein die Erklärung der Anomalie der Periheldrehung beim Merkur auf der Annahme dieser Gleichung, welche im hier relevanten Vakuumfall mit der endgültigen Theorie äquivalent ist [7]. Zu dieser schließlich dringt er eine Woche später in der letzten Mitteilung vor, in der er den Feldgleichungen einen Spurterm hinzufügt, der die Kovarianz der Gleichungen nicht berührt, aber die Einschränkung auf spurfreie Materietensoren aufhebt [6].

Einsteins letzte Note mit den endgültigen, heute sogenannten „Einsteingleichungen“ trägt als Datum der Mitteilung an die Berliner Akademie den 25. November 1915 und wurde eine Woche später ausgegeben. Von Hilbert gibt es nun etwa zeitgleich die von Blumenthal erwähnte Abhandlung mit dem Titel „Die Grundlagen der Physik“, die in den Nachrichten der Gesellschaft der Wissenschaften zu Göttingen erschien [I I]. Die publizierte Fassung trägt als Datum der Mitteilung an die Göttinger Akademie den 20. November 1915, ausgegeben wurde das Heft Ende März 1916, Sonderdrucke waren Mitte Februar erhältlich. Diese Daten und Umstände sind für eine angemessene Einschätzung des Hilbertschen Beitrags wichtig.

In Hilberts gedruckter Arbeit finden wir ebenfalls be- 
reits die korrekten Feldgleichungen der allgemeinen Relativitätstheorie, hergeleitet und formuliert mit Hilfe eines axiomatisch postulierten Variationsprinzips. Hilbert behauptet außerdem, dass seine variationstheoretisch formulierten Gleichungen mit den expliziten Gleichungen aus Einsteins erst fünf Tage später vorgelegten und von Hilbert zitierten Arbeit äquivalent seien.

Ein 1997 erschienener Aufsatz wies darauf hin, dass es Druckfahnen zu Hilberts Mitteilung gibt, die einen Stempel der Setzerei vom 6. Dezember 1915 tragen und die in wesentlichen Punkten von der publizierten Fassung abweichen [5]. Insbesondere enthalten sie noch nicht den Verweis auf Einsteins explizite Gleichungen, und sie schränken für die physikalische Gültigkeit der Theorie die allgemeine Kovarianz noch durch ein Axiom ein, das dem Argument der Einsteinschen Lochbetrachtung ähnelt und in der publizierten Fassung nicht mehr enthalten ist. Diese Entdeckung stellte die vordergründig auf der Basis der Daten der publizierten Arbeiten sich ergebende Chronologie der Einsichten Hilberts und Einsteins infrage und löste damit eine intensive Debatte über das Verhältnis der Hilbertschen Arbeit zu den Einsteinschen Untersuchungen aus $[3,12-15,18]$. Ungeachtet der substanziellen Unterschiede zwischen den Korrekturbögen und der publizierten Fassung enthalten die Korrekturbögen aber bereits die axiomatisch postulierte, allgemein kovariante Variationsformulierung. Es bleibt daher dabei, dass Hilbert damit, wie es Blumenthal ausdrückt, das „Hamiltonsche Prinzip“ (wie es damals genannt wurde) in die allgemeine Relativitätstheorie einführte. Entscheidend für diese Einschätzung ist die Frage, ob bereits in den Korrekturbögen der Riemannsche Krümmungsskalar im Wirkungsintegral auftritt, worauf nämlich die Äquivalenz der Hilbertschen Variationsformulierung mit den expliziten Einsteingleichungen wesentlich beruht.

Etwas kompliziert wird die Rekonstruktion durch die Tatsache, dass das Wirkungsintegral in den Korrekturbögen nicht vollständig spezifiziert ist, da an einem Blatt dieser Bögen ein kleiner Teil fehlt. Es lässt sich aus dem vorhandenen Text allerdings mit einiger Sicherheit rekonstruieren, dass auf dem fehlenden Stück genau der Teil steht, der die Theorie mit Einsteins Theorie äquivalent werden lässt, wenn man von dem außerdem noch vorhandenen zusätzlichen Axiom einmal absieht [16].

Bei dieser verwickelten Quellensituation bleibt, wie gesagt, Blumenthals kurze Einschätzung bei aller Qualifikation, die eine ausführliche Darstellung heute erfordert, erstaunlich präzise. Sie ist auch darin zutreffend, dass neben der Einführung des Hamiltonschen Prinzips die ,Einführung einer Weltfunktion zur Verknüpfung der Gravitation und der elektromagnetischen Erscheinungen" besonders hervorgehoben wird. Hierin unterscheidet sich Hilberts Beitrag in der Tat auch aus heutiger Perspektive noch von Einsteins gleichzeitigen Überlegungen. Diese Formulierung spiegelt aber auch das Physikverständnis der frühen zwanziger Jahre wider, in dem das Programm einer vereinheitlichten Feldtheorie von Gravitation und
Elektromagnetismus für viele Theoretiker, Einstein eingeschlossen, hohe Priorität und Anziehungskraft hatte.

\section{Literatur}

[1] Otto Blumenthal. David Hilbert. Die Naturwissenschaften, 10:67-72, 1922

[2] Max Born. Hilbert und die Physik. Die Naturwissenschaften, 10:88-93, 1922.

[3] Katherine Brading and Tom Ryckman. Hilbert's 'Foundations of Physics': Gravitation and Electromagnetism within the Axiomatic Method. Studies in the History and Philosophy of Modern Physics, 39:102-153, 2008.

[4] Leo Corry. David Hilbert and the Axiomatization of Physics (1898-1918). From Grundlagen der Geometrie to Grundlagen der Physik. Dordrecht: Kluwer Academic, 2004.

[5] Leo Corry, Jürgen Renn, and John Stachel. Belated decision in the Hilbert-Einstein priority dispute. Science, 278:1270-1273, 1997.

[6] Albert Einstein. Die Feldgleichungen der Gravitation. Königliche Preußische Akademie der Wissenschaften. Sitzungsberichte, pages 844-847, 1915.

[7] Albert Einstein. Erklärung der Perihelbewegung des Merkur aus der allgemeinen Relativitätstheorie. Königliche Preußische Akademie der Wissenschaften. Sitzungsberichte, pages 83I-839, 1915.

[8] Albert Einstein. Zur allgemeinen Relativitätstheorie. Königliche Preußische Akademie der Wissenschaften. Sitzungsberichte, pages 778-786, 1915.

[9] Albert Einstein. Zur allgemeinen Relativitätstheorie (Nachtrag). Königliche Preußische Akademie der Wissenschaften. Sitzungsberichte, pages 799-801, 1915.

[10] Albert Einstein and Marcel Grossmann. Entwurf einer verallgemeinerten Relativitätstheorie und einer Theorie der Gravitation. Zeitschrift für Mathematik und Physik, 62:225-26I, 1913.

[II] David Hilbert. Die Grundlagen der Physik. (Erste Mitteilung.). Königliche Gesellschaft der Wissenschaften zu Göttingen. Math.phys. Klasse. Nachrichten, pages 395-407, 1915.

[12] A.A. Logunov, M.A. Mestvirishvili, and V.A. Petrov. How were the Hilbert-Einstein equations discovered? Physics-Uspekhi, 2004:607-621, 2004.

[13] Jürgen Renn and John Stachel. Hilbert's Foundation of Physics: From a Theory of Everything to a Constituent of General Relativity. In Jürgen Renn, editor, The Genesis of General Relativity, Vol. 4, pages 857-974. : Springer, 2007.

[14] David E. Rowe. Einstein Meets Hilbert: At the Crossroads of Physics and Mathematics. Perspective on Physics, 3:379-424, 2001.

[15] Tilman Sauer. The Relativity of Discovery: Hilbert's First Note on the Foundations of Physics. Archive for History of Exact Sciences, 53:529-575, 1999.

[16] Tilman Sauer. Einstein Equations and Hilbert Action: What is missing on page 8 of the proofs for Hilbert's First Communication on the Foundations of Physic? Archive for History of Exact Sciences, 59:577-590, 2005.

[17] Tilman Sauer and Ulrich Majer, editors. David Hilbert's Lectures on the Foundations of Physics, 1915-1927. Dordrecht etc: Springer, 2009.

[18] Vladimir P. Vizgin. On the discovery of the gravitational field equations by Einstein and Hilbert: new materials. PhysicsUspekhi, 44:I283-I298, 200 I.

Dr. Tilman Sauer Einstein Papers Project California Institute of Technology 20-7 I200 E. California Blvd. Pasadena, CA 9II25, USA. tilman@caltech.edu

Prof. David E. Rowe, Johannes Gutenberg-Universität, Institut für Mathematik, Staudingerweg 9, 55099 Mainz. rowe@mathematik. uni-mainz.de 